\title{
Dynamic Modelling of an Orientable Solar Panel System as a 2-DOF Manipulator
}

\author{
Modelado Dinámico de un Sistema Solar de Panel Orientable como un \\ Manipulador de 2 Grados de Libertad
}

\author{
A. Fernández-Pizarro (iD ; J. Beltrán-González (iD ; J. Roldán-Mckinley
}

\begin{abstract}
Lagrange principle is used to derive the dynamic equation of an Orientable Solar Panel System-OSPS. Prior to the dynamic formulation, the OSPS kinematic analysis was eased by considering the system as a 2 degrees-of-freedom open-loop serial manipulator with perpendicular revolute joints. DenavitHartenberg parameters let finding the transformation matrix to relate the solar panel with the fixed element was stated. Then, existing formulation about dynamics for serial manipulators was adapted to the OSPS. The explicit dynamic model was used to build a Simunlink block. Another plant was created in SimMechanics. Then, performance of both plants when using the same PID controller were compared for a typical movement of the OSPS system. Results showed agreement for both cases, suggesting that the dynamic model is suitable for further simulations and implementation.
\end{abstract}

Index Terms-Manipulator Dynamics, OSPS, 2R open-loop.

Resumen-El principio de Lagrange es usado para derivar la ecuación dinámica de un Sistema Solar de Panel Solar Orientable (OSPS por sus siglas en inglés de Orientable Solar Panel System). Previo a la formulación dinámica, el análisis cinemático de facilitó mediante la consideración del sistema como un manipulador serial de cadena abierta de dos grados de libertad con dos juntas de revoluta. Los parámetros Denavit-Hartenberg permitieron encontrar la matriz de transformación para relacionar el panel solar con la tierra o bastidor. Luego, la formulación existente acerca de dinámica para manipuladores seriales fue aplicada ala sistema solar con panel orientable. El modelo dinámico explícito fue usado para construir un bloque de Simulink. Otra planta fue creada en SimMechanics. Luego, el desempeño de ambas plantas ante un mismo controlador PID fue comparado para un movimiento típico del sistema solar de panel

This manuscript was sent on January 24, 2019 and accepted on June 26, 2019.

This work was supported by Research Affairs Division (Vicerrectoría de Investigaciones) of Universidad del Atlántico (Puerto Colombia, Colombia).

J. Roldán-Mckinley works currently in the Mechanical Engineering Program (DIMER research group) at Universidad del Atlántico (Puerto Colombia, Colombia) (e-mail: javierroldan@mail.uniatlantico.edu.co).

A. Fernández-Pizarro is a Mechanical Engineer from Universidad del Atlántico (Puerto Colombia, Colombia). He works as a Maintenance Engineer at Tubos 360 Company (Caracolí, Colombia) (e-mail: andres.fernandez@t360.com, affernandez@mail.uniatlantico.edu.co).

J. Beltrán-González is a Mechanical Engineer from Universidad del Atlántico (Puerto Colombia, Colombia). He works as an Operations Engineer at Navarro Tovar SAS Company (Barranquilla, Colombia) (e-mail: operaciones@navarrotovar.com.co,jabeltran@mail.uniatlantico.edu.co). orientable. Los resultados mostraron concordancia para ambos casos, sugiriendo que el modelo dinámico es adecuado para futuras simulaciones e implementación.

Palabras claves - Dinámica de manipulador, OSPS, 2R cadena abierta

\section{INTRODUCTION}

$\mathrm{E}$ LECTRIC generation output is greater in Orientable Solar Panel Systems (OSPS) where the sun rays incidence is kept perpendicular to the panel surface. Comparison studies between fixed and OSPS with two rotation axes, show that the latter is about 20 to $40 \%$ more efficient [1], depending on the weather conditions, the season of the year, and geographic location where the system is located at [2]. The right orientation of the system will greatly depend on the dynamic model of the system in order to implement the appropriate control strategy. The most OSPS track the sun with photoresistor based sensors that are located on extreme sides of the panel; however, the problem becomes more interesting when the dynamic model of the OSPS is considered, given the non-linearity involved.

In 2016, Kumar and Sharma [3] use Proportional Integral Derivative-PID technique to improve maximum power and maximum voltage but only considering the electric circuit of the system. The dynamic model of the physical system was not considered. Similarly, a controller that combines Internal Model Control-IMC, also with PID, is presented in [4] for improving the speed response of a dual-axis solar panel system. No dynamic model of the plant was presented. Also in the same year, Kiyak and Gol [5] compare fuzzy logic and PID controllers to find the perpendicular reflection of the sun rays to a solar panel. Later, Dwivedi and Saket [6] used a PID controller for improving the performance of the voltage and peak power of a single axis panel. However, they did not modelled the dynamic of the physical system. Also without an explicit dynamic model of the plant, Safan et al. [7] evaluate the accuracy of a sun tracking system with a Multi-Degree of Freedom-Simplified Universal Intelligent PID controller (MDOF-SUI PID).

More recently, a direct formula is proposed in [8] for the design of a robust PID controller for sun tracker system using 
quadratic regulator approach with compensating pole (QRAWCP). They model the DC servo motor and the gear reducer but did not show the dynamic model of the two-axis solar system. For OSPS modelling, the most of the reported cases use specialized multibody software. Alexandru et al $[9,10]$, models the OSPS Dynamics with a SolidWorks' CAD that is exported to MSC ADAMS and later to SimMechanics.

In this paper, the dynamic model of an OSPS is obtained. As a novelty, the OSPS is analyzed like a 2 Degrees-OfFreedom (DOF) open chain manipulator with perpendicular consecutive axis. The equivalent manipulator is comprised by two revolute joints, thus achieving the elevation and azimuth motions required for OSPS. This approach simplifies the kinematic and the dynamic modelling since existing literature for 2-DOF revolute serial manipulators can be rapidly adapted. The developed analytical plant is compared with another plant from SimMechanics (Matlab). Then, the same PID controller is used to compare performance of each plant.

\section{MATERIALS AND METHODS}

\section{A. Kinematic Model}

A systematic robot labelling notation widely used is the Denavit-Hartenberg parameters [11]. Two angles and two distances allow establishing recurrence. Fig. 1.a shows the three links that comprises the representation of the 2-DOF OSPS: link 0 or grounded element, link 1 for azimuth motion, and link 2 for elevation motion.

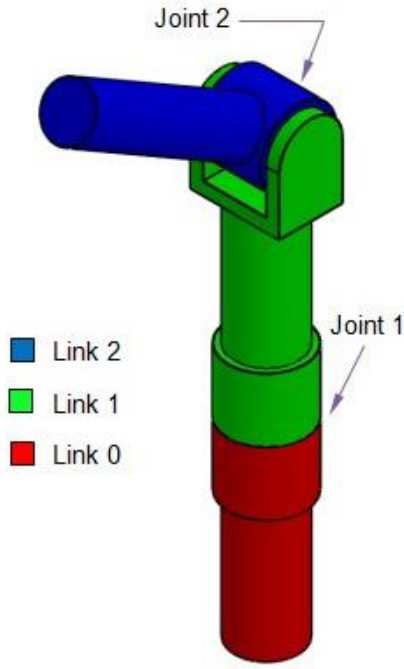

a. OSPS elements

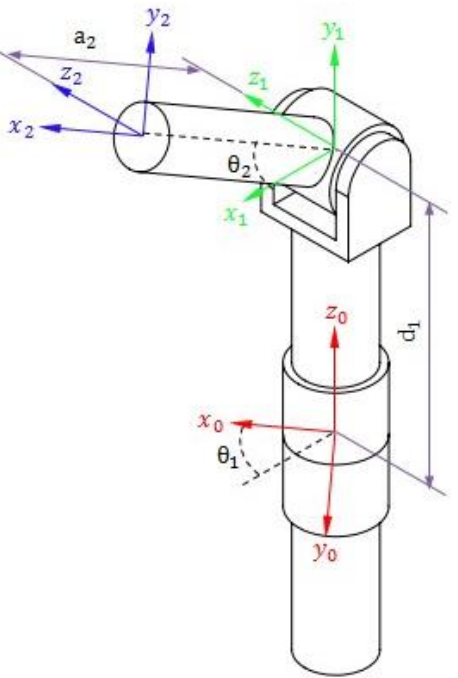

b. Denavit-Hartenberg parameters
Fig. 1. Denavit-Hartenberg parameters of the OSPS.

Fig. 2 depicts the azimuth and elevation motion of the panel. Each element has a reference system, Fig. 1.b: fixed (inertial) $\left\{\mathrm{x}_{0}-\mathrm{y}_{0}-\mathrm{Z}_{0}\right\}$ or $\{0\}$, movable reference system $\left\{\mathrm{x}_{1}-\mathrm{y}_{1}-\mathrm{z}_{1}\right\}$ or $\{1\}$ for the azimuth motion, and local reference system $\left\{\mathrm{x}_{2}-\mathrm{y}_{2}-\mathrm{z}_{2}\right\}$ or $\{2\}$ for the elevation motion. The latter element is attached to the panel and the securing structure elements.

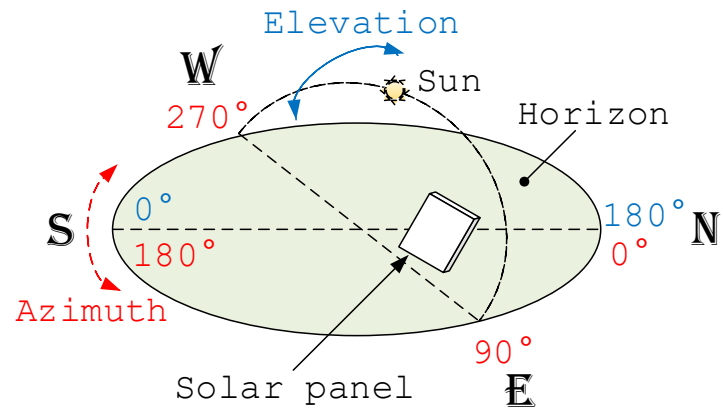

Fig. 2. Azimuth and elevation references for OSPS panel.

Fig. 1.b also depicts the Denavit-Hartenberg parameters. Joint angle parameter $\theta_{i}$ is the angle that element $i$-th must be rotated about the $\mathrm{z}_{\mathrm{i}-1}$ axis in order to align axes $\mathrm{x}_{\mathrm{i}-1}$ and $\mathrm{x}_{\mathrm{i}}$ in the same plane, while axis $y_{i-1}$ and $y_{i}$ have the same direction. Distance $d_{i}$ is the offset distance along the $z_{i-1}$ axis from the origin of the (i-1)-th system $\{\mathrm{i}-1\}$ to the origin of the system $\{\mathrm{i}\}$. Distance $\mathrm{a}_{\mathrm{i}}$ is the offset distance along the $\mathrm{x}_{\mathrm{i}-1}$ axis from the origin of system $\{i-1\}$ to the origin of the system $\{i\}$. Angle $\alpha_{\mathrm{i}}$ is the angle about the $\mathrm{x}_{\mathrm{i}-1}$ axis that the must be rotated the system $\{\mathrm{i}-1\}$ to get aligned the axes $\mathrm{z}_{\mathrm{i}-1}$ and $\mathrm{z}_{\mathrm{i}}$, after turning the angle $\theta_{\mathrm{i}}$. The recurrence in the parameters leads to a transformation matrix, ${ }^{\mathrm{i}-1} \mathbf{A}_{\mathrm{i}}$, as the result of successive standard transformations to relate two consecutive coordinate axes $\{i-1\}$ and $\{i\}$, given by (1) [12], where $c_{i}$ and $s_{i}$ are the cosine and sine of $\theta_{\mathrm{i}}$, respectively; and $\mathrm{c}_{\alpha \mathrm{i}}$ and $\mathrm{s}_{\mathrm{\alpha i}}$ are the cosine and sine of the link angle $\alpha_{\mathrm{i}}$, respectively. Table 1 presents the numerical values [13] of the Denavit-Hartenberg parameters of the OSPS in Fig. 1.b. From the recurrence in (1) is possible to relate the coordinate systems $\{0\},\{1\}$ and $\{2\}$ to yield the transformation matrix of the OSPS that relates the second element with the ground, as presented in (2) $[11,12,14]$. For the case in this report, the parameter values are $\mathrm{d}_{1}=355 \mathrm{~mm}, \mathrm{~d}_{2}=0 \mathrm{~mm}, \mathrm{a}_{1}=0 \mathrm{~mm}, \mathrm{a}_{2}=91 \mathrm{~mm}, \alpha_{1}=90^{\circ}, \alpha_{2}=0^{\circ}$.

$$
\begin{gathered}
{ }^{\mathrm{i}-1} \mathbf{A}_{\mathrm{i}}=\left[\begin{array}{cccc}
\mathrm{c}_{\mathrm{i}} & -\mathrm{c}_{\alpha \mathrm{i}} \mathrm{s}_{\alpha \mathrm{i}} & \mathrm{s}_{\alpha \mathrm{i}} \mathrm{s}_{\mathrm{i}} & \mathrm{a}_{\mathrm{i}} \mathrm{c}_{\mathrm{i}} \\
\mathrm{s}_{\mathrm{i}} & \mathrm{c}_{\alpha \mathrm{i}} \mathrm{c}_{\mathrm{i}} & -\mathrm{s}_{\alpha \mathrm{i}} \mathrm{c}_{\mathrm{i}} \mathrm{a}_{\mathrm{i}} \mathrm{s}_{\mathrm{i}} \\
0 & \mathrm{~s}_{\mathrm{i}} & \mathrm{c}_{\mathrm{i}} & \mathrm{d}_{\mathrm{i}} \\
0 & 0 & 0 & 1
\end{array}\right] \\
{ }^{0} \mathbf{A}_{2}={ }^{0} \mathbf{A}_{1}{ }^{1} \mathbf{A}_{2}=\left[\begin{array}{cccc}
\mathrm{c}_{1} \mathrm{c}_{2} & -\mathrm{c}_{1} \mathrm{~s}_{2} & \mathrm{~s}_{1} & \mathrm{a}_{2} \mathrm{c}_{1} \mathrm{c}_{2} \\
\mathrm{~s}_{1} \mathrm{c}_{2} & -\mathrm{s}_{1} \mathrm{~s}_{2} & -\mathrm{c}_{1} & \mathrm{a}_{2} \mathrm{~s}_{1} \mathrm{c}_{2} \\
\mathrm{~s}_{2} & \mathrm{c}_{2} & 0 & \mathrm{a}_{2} \mathrm{~s}_{2}+\mathrm{d}_{1} \\
0 & 0 & 0 & 1
\end{array}\right]
\end{gathered}
$$

\section{B. Dynamic Model}

Stating the dynamic equation of a manipulator allows relating applied forces at the actuated joints with the expected motion [15]. In the direct dynamics analysis, the equation of motion is used to find the response of the robot arm to some applied forces. In the inverse dynamics problem, this equation is used to find the actuator torques and/or forces required to generate a desired path at the tool $[12,15]$. Three approaches are used for establishing the dynamic equation of a manipulator: Newton-Euler, virtual work principle, and 
Lagrange formulation $[12,15,16]$. The dynamic model of serial manipulators is found in a simpler way by the Lagrangian method [16]. The general formulation presented in [16] is adapted next for the 2-DOF serial manipulator that represents the OSPS. The Langrange function, $\mathbf{L}$, of the robot is found by (3), with K: kinetic energy, $\mathbf{U}$ : potential energy of the manipulator (OSPS). Equation (4) shows the time evolution of the OSPS as a function of the $\mathrm{n}$ generalized coordinates, with $\mathrm{q}_{\mathrm{i}}$ : i-th generalized coordinate, Qi: i-th generalized force of the system ( $\mathrm{i}=1,2$ since there are 2 revolute joints) or torques applied at each joint by the motors. Equation (5) describes the dynamics of the system, with $\tau$ : applied torque vector at the joints, $\mathbf{M}$ : inertia matrix, $\ddot{\mathbf{q}}$ : angular acceleration vector, $\mathbf{V}$ : centrifugal and Coriollis forces vector, and $\mathbf{G}$ : torque due to the gravity effect. The kinetic energy of the $\mathrm{i}$-th element, $\mathrm{K}_{\mathrm{i}}$, is given by (6), where the contribution of both center of mass linear velocity, $\mathbf{v}_{\mathrm{ci}}$, and angular velocity, $\boldsymbol{\omega}_{\mathrm{i}}$, of $\mathrm{i}$-th element is considered with $\mathrm{m}_{\mathrm{i}}$ : mass of the element, and $\mathbf{I}_{\mathrm{i}}$ : inertia matrix of the element with respect to its own center of mass. Term $\mathbf{I}_{\mathrm{i}}$ is found by (7), where ${ }^{0} \mathbf{R}_{\mathrm{i}}$ is the orientation matrix of the element as seen if the fixed coordinate system $\{0\}$ given by the $3 \times 3$ submatrix $[(1,1) \ldots(3,3)]$ of the transformation matrix in (2); $\mathrm{m}_{\mathrm{i}}$ is the mass of the element; and ${ }^{\mathrm{i}} \mathbf{I}_{\mathrm{i}}$ is the inertia tensor in its own center of mass, as seen in its own coordinate system $\{\mathrm{i}\}$, as seen in (8).

$$
\begin{aligned}
& L=K-U \\
& Q_{i}=\frac{d}{d t}\left(\frac{\partial L}{\partial \dot{q}_{i}}\right)-\frac{\partial L}{\partial q_{i}} \\
& \tau=M \ddot{q}+V+G \\
& \mathbf{K}_{\mathrm{i}}=\frac{1}{2} \mathbf{v}_{\mathrm{ci}}^{\mathrm{T}} \mathrm{m}_{\mathrm{i}} \mathbf{v}_{\mathrm{ci}}+\frac{1}{2} \boldsymbol{\omega}_{\mathrm{i}}^{\mathrm{T}} \mathrm{I}_{\mathrm{i}} \boldsymbol{\omega}_{\mathrm{i}} \\
& \mathbf{I}_{\mathrm{i}}={ }^{0} \mathbf{R}_{\mathrm{i}}{ }^{\mathrm{i}} \mathbf{I}_{\mathrm{I}}\left[{ }^{0} \mathbf{R}_{\mathrm{i}}\right]^{\mathrm{T}} \\
& { }^{\mathrm{i}} \mathrm{I}_{\mathrm{i}}=\mathrm{m}_{\mathrm{i}}\left[\begin{array}{ccc}
\mathrm{I}_{\mathrm{i} 1} & 0 & 0 \\
0 & \mathrm{I}_{\mathrm{i} 2} & 0 \\
0 & 0 & \mathrm{I}_{\mathrm{i} 3}
\end{array}\right]
\end{aligned}
$$

The total velocity vector of the center of mass of the i-th element, $\dot{\mathbf{x}}_{\mathrm{ci}}$, given by (9), is related with the generalized coordinates velocity vector, $\dot{\mathbf{q}}$, by the Jacobian matrix of the element, $\mathbf{J}_{\mathrm{i}}$, as seen in (10). The $6 \times 1$ size Jacobian matrix of the element is created from the two $3 \times 1$ submatrices, see (11), $\mathbf{J}_{\mathrm{vi}}$ and $\mathbf{J}_{\omega \mathrm{i}}$ that represent the center of mass linear velocity Jacobian and angular Jacobian matrixes of the element, respectively. Submatrices $\mathbf{J}_{\mathrm{vi}}$ and $\mathbf{J}_{\boldsymbol{~} \mathrm{i}}$ for revolute joints are found from the recurrence in (12) and (13), respectively, where $\mathbf{z}_{\mathbf{j}-1}$ : unit vector for the direction of the rotation axis of the element $j-1$, as seen in $\{0\} ;{ }^{j-1} \mathbf{p}_{\mathrm{ci}}$ : position vector from the origin of $\{j-1\}$ to the center of mass of the $\mathrm{i}$-th element, and the " $x$ " in (12) indicates the cross product operation. The size of submatrices $\mathbf{J}_{\mathrm{vi}}$ and $\mathbf{J}_{\omega \mathrm{i}}$ is $3 \times n$, when $\mathrm{j}>\mathrm{i}$ these entities are completed with $[\mathbf{0}]_{1 \times n}$ as seen in (14) and (15), respectively, where $\mathrm{n}$ is the number of generalized coordinates. The inertia matrix, M, of the system is found with (16). The total potential energy of the OSPS, $\mathbf{U}$, is also found with (17), where $\mathbf{g}^{\mathrm{T}}$; column gravity vector with $\mathrm{g}=9.81 \mathrm{~m} / \mathrm{s}^{2}$.

$$
\begin{aligned}
& \dot{\mathbf{x}}_{\mathrm{ci}}=\left[\begin{array}{c}
\mathbf{v}_{\mathrm{ci}} \\
\boldsymbol{\omega}_{\mathrm{i}}
\end{array}\right] \\
& \dot{\mathbf{x}}_{\mathrm{ci}}=\mathbf{J}_{\mathrm{i}} \dot{\mathbf{q}} \\
& \mathbf{J}_{\mathrm{i}}=\left[\begin{array}{l}
\mathbf{J}_{\mathrm{vi}} \\
\mathbf{J}_{\omega \mathrm{i}}
\end{array}\right] \\
& \mathbf{J}_{v i}^{\mathrm{j}}=\left[\mathbf{z}_{\mathrm{j}-1} \times^{\mathrm{j}-1} \mathbf{p}_{\mathrm{ci}}\right] \\
& \mathbf{J}_{\omega \mathrm{i}}^{\mathrm{j}}=\left[\mathbf{z}_{\mathrm{j}-1}\right] \\
& \mathbf{J}_{\mathrm{vi}}=\left[\begin{array}{llllll}
\mathbf{J}_{\mathrm{vi}}^{1} & \mathbf{J}_{\mathrm{vi}}^{2} & \ldots . \mathbf{J}_{\mathrm{vi}}^{\mathrm{i}} & \mathbf{0} & \ldots & \mathbf{0}
\end{array}\right]
\end{aligned}
$$

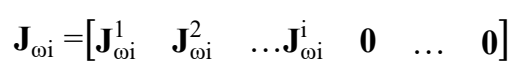

$$
\begin{aligned}
& \mathbf{M}=\sum_{\mathrm{i}=1}^{\mathrm{n}}\left(\mathbf{J}_{\mathrm{vi}}^{\mathrm{T}} \mathrm{m}_{\mathrm{i}} \mathbf{J}_{\mathrm{vi}}+\mathbf{J}_{\omega \mathrm{i}}^{\mathrm{T}} \mathbf{I}_{\mathrm{i}} \mathbf{J}_{\omega \mathrm{i}}\right) \\
& \mathbf{U}=-\sum_{\mathrm{i}=1}^{\mathrm{n}}\left(\mathrm{m}_{\mathrm{i}} \mathbf{g}^{\mathrm{T}} \mathbf{p}_{\mathrm{ci}}\right) ; \mathbf{g}^{\mathrm{T}}=\left[\begin{array}{lll}
0 & 0 & -\mathrm{g}
\end{array}\right]^{\mathrm{T}}
\end{aligned}
$$

The i-th term of the centrifugal and Coriolis forces vector, $\mathbf{V}_{\mathrm{i}}$, is obtained with (18), where the forces vector is given in (19). The torque contribution of each element's weight, $\mathbf{G}_{\mathrm{i}}$, yields (20), the total vector is also given in (21). Similarly, the external torque elements, $\boldsymbol{\tau}_{\mathrm{i}}$, let yielding the external forces and torques vector $\tau$, in (22). By substituting (6) to (17) into (3) and solving into (4), it is obtained the dynamic equation of the manipulator. For the inverse dynamics, it is solved from (5) to yield (23). By following the presented formulation it is obtained the dynamic model for the OSPS in (24). The attained model is non-linear and the torque applied by the motors in both joints will vary along the motion. It is also apparent from the same equation that the inertia to be overcome by the motor at joint 2 is constant. However, inertia load for the motor at joint 1 will change. Since both joints are revolute type (no prismatic joint), there are not Coriolis terms in the model but centrifugal force terms. Due to the design of the OSPS, only the weight of the second element is contributing to the gravity term.

$$
\begin{gathered}
\mathrm{V}_{\mathrm{i}}=\sum_{\mathrm{j}=1}^{\mathrm{n}} \sum_{\mathrm{k}=1}^{\mathrm{n}}\left(\frac{\partial \mathrm{M}_{\mathrm{ij}}}{\partial \mathrm{q}_{\mathrm{i}}}-\frac{1}{2} \frac{\partial \mathrm{M}_{\mathrm{jk}}}{\partial \mathrm{q}_{\mathrm{i}}}\right) \dot{\mathrm{q}}_{\mathrm{j}} \dot{\mathrm{q}}_{\mathrm{k}} \\
\mathbf{V}=\left[\begin{array}{llll}
\mathrm{V}_{1} & \mathrm{~V}_{2} & \ldots & \mathrm{V}_{\mathrm{n}}
\end{array}\right]^{\mathrm{T}}
\end{gathered}
$$




$$
\begin{aligned}
& \mathrm{G}_{\mathrm{i}}=\sum_{\mathrm{j}=1}^{\mathrm{n}} \mathrm{m}_{\mathrm{i}} \mathbf{g}^{\mathrm{T}} \mathrm{J}_{\mathrm{vj}}^{\mathrm{i}} \\
& \mathbf{G}=\left[\begin{array}{llll}
G_{1} & G_{2} & \ldots & G_{n}
\end{array}\right]^{T} \\
& \boldsymbol{\tau}=\left[\begin{array}{llll}
\tau_{1} & \tau_{2} & \ldots & \tau_{n}
\end{array}\right]^{\mathrm{T}} \\
& \ddot{\mathrm{q}}=\mathrm{M}^{-1}(\tau-\mathrm{V}-\mathrm{G}) \\
& {\left[\begin{array}{l}
\tau_{1} \\
\tau_{2}
\end{array}\right]=\left[\begin{array}{cc}
\mathrm{I}_{12}+\mathrm{m}_{2} \mathrm{c}_{\mathrm{g} 2}^{2} \mathrm{c}_{2}^{2}+\mathrm{I}_{21} \mathrm{~s}_{2}^{2}+\mathrm{I}_{22} \mathrm{c}_{2}^{2} & 0 \\
0 & \mathrm{~m}_{2} \mathrm{c}_{\mathrm{g} 2}^{2}+\mathrm{I}_{23}
\end{array}\right]\left[\begin{array}{c}
\ddot{\theta}_{1} \\
\ddot{\theta}_{2}
\end{array}\right]+\ldots} \\
& {\left[\begin{array}{c}
-2 s_{2} c_{2}\left(m_{2} c_{g 2}^{2}-I_{21}+I_{22}\right) \dot{\theta}_{1} \dot{\theta}_{2} \\
s_{2} c_{2}\left(m_{2} c_{g 2}^{2}-I_{21}+I_{22}\right) \dot{\theta}_{1}^{2}
\end{array}\right]+\left[\begin{array}{c}
0 \\
m_{2} g c_{g 2} c_{2}
\end{array}\right]}
\end{aligned}
$$

\section{RESULTS AND DISCUSSION}

A controller was designed to insert in the plant the developed dynamic model, as a way of evaluating the dynamics. It is chosen the classical Proportional Integral Derivative-PID control strategy as shown in the Simulink model in Fig. 3. The equivalent Simulink block diagram is depicted in Fig. 4. The reference position, velocity and acceleration are $\mathbf{q}_{\mathrm{d}}, \dot{\mathbf{q}}_{\mathrm{d}}$ and $\ddot{\mathbf{q}}_{\mathbf{d}}$, respectively. The controller gains are set as $\mathbf{K}_{\mathrm{p}}=20 \mathbf{I}_{2 \times 2}, \mathbf{K}_{\mathrm{i}}=0 \mathbf{I}_{2 \times 2}$ and $\mathbf{K}_{\mathrm{d}}=10 \mathbf{I}_{2 \times 2}$. Fig. 5 presents the OSPS or plant Simulink model from (24), and Fig. 6 depicts a SimMechanics model for the plant. Table 1 presents the numerical dynamic parameters used for the plant [13]. For the simulation, the OSPS location is set at Universidad del Atlántico soccer field (Puerto Colombia, Colombia), latitude: 11.0159731 , longitude: -4.8746478 , date January 1st, 2017 (from www.SunEarthTools.com [17]). It is simulated the las motion or the end of day cycle, at 19:00 PM.

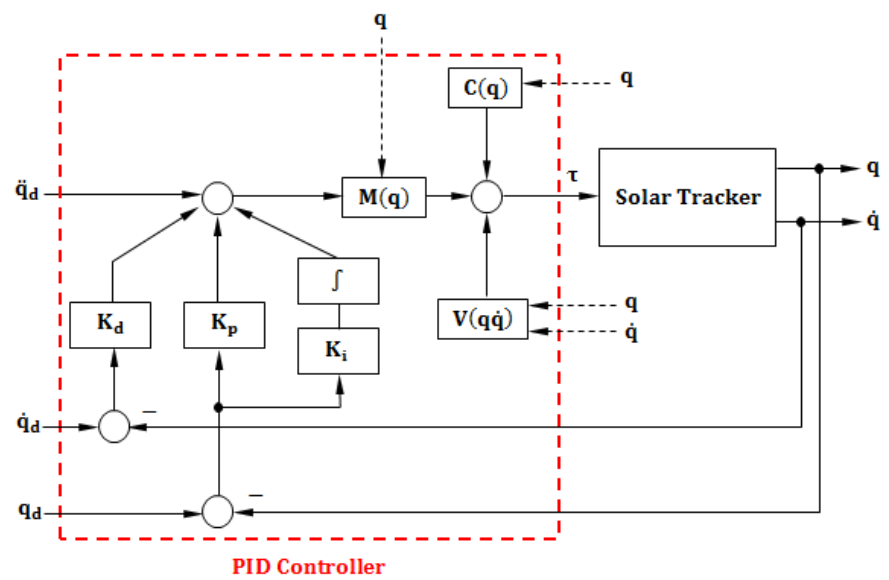

Fig. 3. PID control scheme for the OSPS.

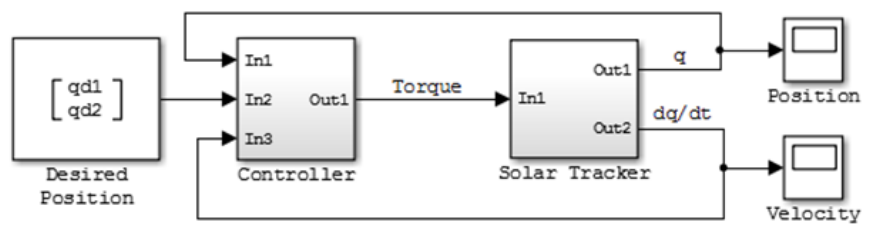

Fig. 4. Simulink model of the control law.

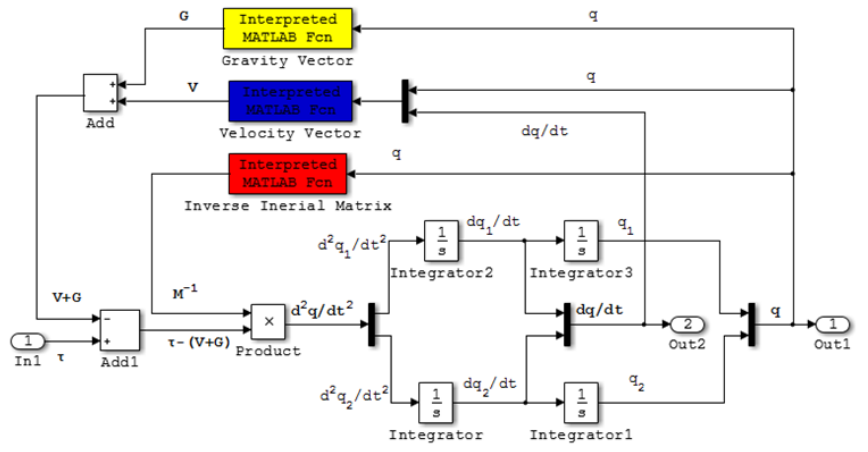

Fig. 5. Simulink model for the OSPS from (24).

The begin and end OSPS positions are $(-244,9.9)^{\circ}$ and ($115.5,9.9)^{\circ}$ [17], respectively, (Azimuth, Elevation). From results in Fig. 7, it is observed the same torques are obtained along time for each joint. It is to say, by using the same controller for both plants, analytical plant versus SimMechanics plant, the obtained results are similar for each case. This result is considered satisfactory for the developed

\begin{tabular}{|c|c|c|c|c|c|}
\hline \multicolumn{5}{|c|}{ Parameter } & Value \\
\hline \multirow{2}{*}{$\begin{array}{l}\text { Inertia } \\
\left(\mathrm{kg} \cdot \mathrm{m}^{2}\right)\end{array}$} & ${ }^{1} I_{1}=$ & {$\left[\begin{array}{r}\mathrm{I}_{11} \\
0 \\
0\end{array}\right.$} & $\begin{array}{c}0 \\
\mathrm{I}_{12} \\
0\end{array}$ & $\left.\begin{array}{c}0 \\
0 \\
\mathrm{I}_{13}\end{array}\right]$ & $\begin{array}{l}\mathrm{I}_{11}=0.05026 \\
\mathrm{I}_{12}=0.00679 \\
\mathrm{I}_{13}=0.05006\end{array}$ \\
\hline & ${ }^{2} I_{2}=$ & {$\left[\begin{array}{r}\mathrm{I}_{21} \\
0 \\
0\end{array}\right.$} & $\begin{array}{c}0 \\
I_{22} \\
0\end{array}$ & $\left.\begin{array}{c}0 \\
0 \\
\mathrm{I}_{23}\end{array}\right]$ & $\begin{array}{l}\mathrm{I}_{21}=11.36103 \\
\mathrm{I}_{22}=3.02971 \\
\mathrm{I}_{23}=8.42094\end{array}$ \\
\hline Mass (kg) & \begin{tabular}{|l|} 
Joint 1 \\
Joint 2
\end{tabular} & & & & \begin{tabular}{|l|}
4.23619 \\
37.73207
\end{tabular} \\
\hline$c_{g i}(\mathrm{~mm})$ & \begin{tabular}{|l|} 
Joint 1 \\
Joint 2
\end{tabular} & & & & \begin{tabular}{|l}
-170.08 \\
-28.95
\end{tabular} \\
\hline
\end{tabular}
model, although further simulations are suggested.

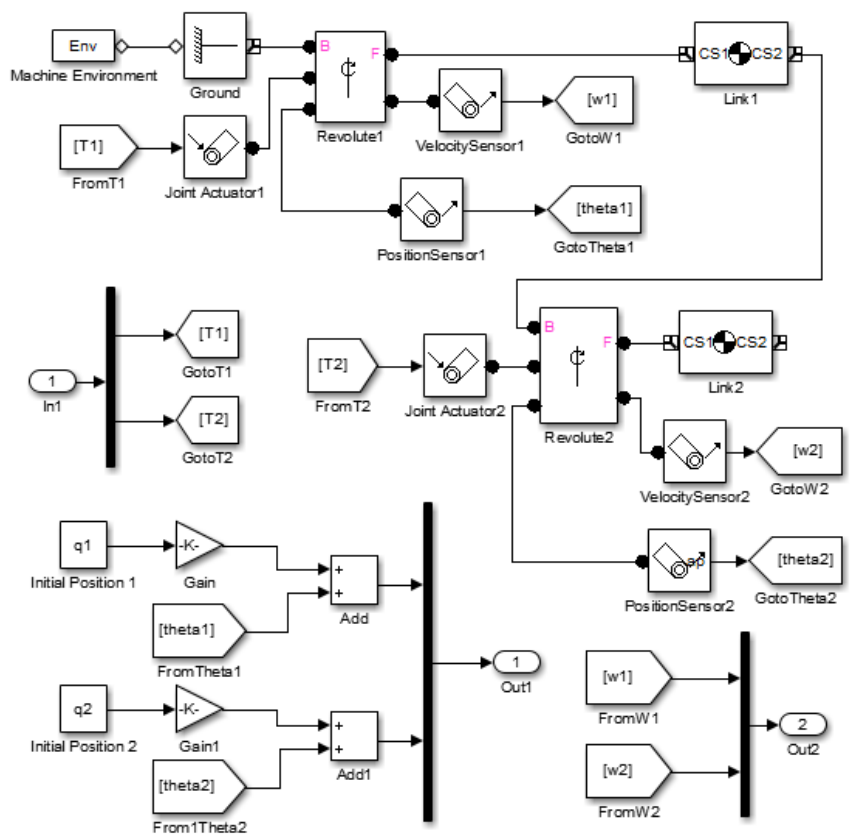

Fig. 6. SimMechanics model of the plant inserted in the Simulink file.

TABLE I

PARAMETERS FOR THE DYNAMIC MODEL 


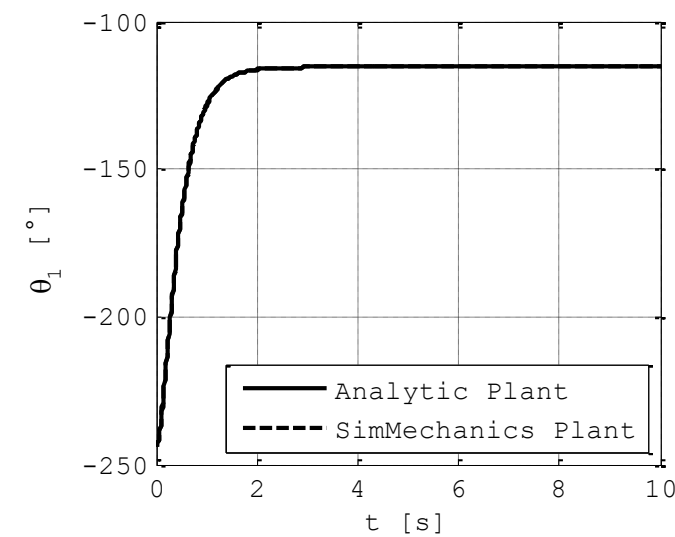

a. Joint angle $\theta_{1}$ for joint 1 , Azimuth

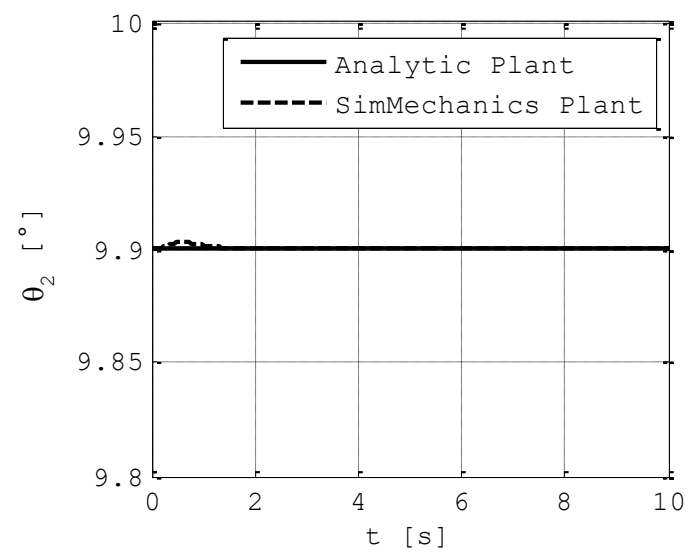

b. Joint angle $\theta 2$ for joint 2, Elevation

Fig. 7. Azimuth and elevation joint angles for the simulation.

\section{CONCLUSION}

The main contribution of this paper was the modelling of an OSPS as a 2-DOF open-loop serial manipulator. This approach eased developing the kinematic and the dynamic models of the OSPS plant. A transformation matrix to relate the solar panel (tool) with the ground was derived with the Denavit-Hartenberg parameters. Later, the Lagrangian formulation was used to generate the OSPS inertia matrix, centrifugal torques vector and the gravitational vector for setting the dynamic equation of the system. The analytical model was compared with the parameters of a SimMechanics plant. A single PID controller was used for both plants to compare the performance of each joint, as a way of contrasting the dynamic models. With the proposed methodology for modelling the OSPS, it is expected to facilitate the control strategies implementation for properly orienting the solar panel perpendicular to the sun rays.

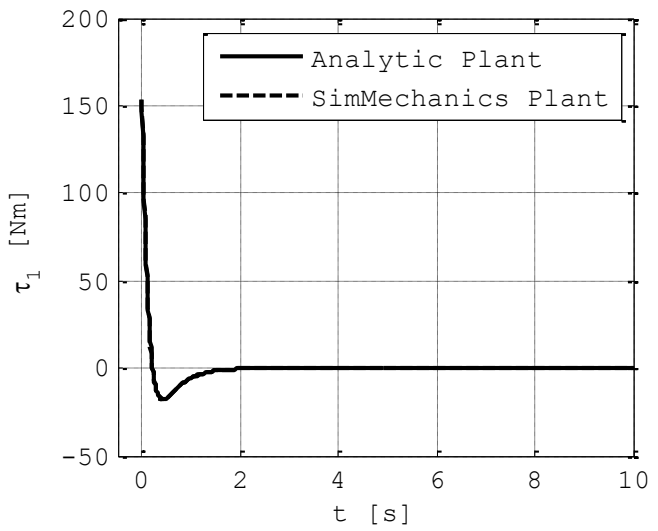

a. Torque for joint 1, Azimuth

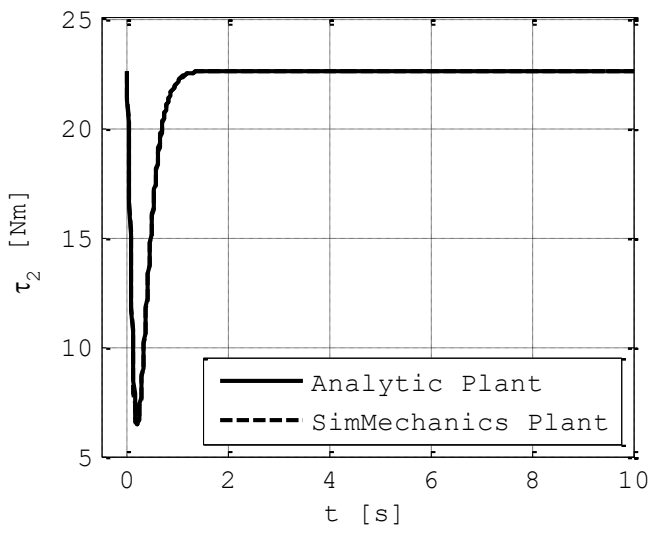

b. Torque for joint 2, Elevation

Fig. 8. Azimuth and elevation joint torques for the simulation.

\section{REFERENCES}

[1] R. Eke, and A. Senturk, "Performance comparison of a double-axis sun tracking versus fixed PV system," Solar Energy, vol. 86, no. 9, pp. 2665-2672, 2012. DOI: 10.1016/j.solener.2012.06.006

[2] M. Koussa, M. Haddadi, D. Saheb, A. Malek, and S. Hadji, "Sun tracker systems effects on flat plate photovoltaic PV systems performance for different sky states: A case of an arid and hot climate," Energy Procedia, vol. 18, pp. 839-850, 2012. DOI: 10.1016/j.egypro.2012.05.098

[3] N. Kumar, and N. Sharma, "Improve performance of PV system by PID controller," Int. J. of Science, Engineering and Technology, vol. 4, no. 1, pp. 227-232, 2016.

[4] B. Oladayo, and A. Titus, "Development of solar tracking system using IMC-PID controller," American J. of Engineering Research, vol. 5, no. 5, pp. 135-142, 2016.

[5] E. Kiyak, and G. Gol, "A comparison of fuzzy logic and PID controller for a single-axis solar tracking system," Renewables: Wind, Water, and Solar, vol. 3, no. 7, 14 p, 2016. DOI: 10.1186/s40807-016-0023-7

[6] L. Dwivedi, and R. Saket, "Improve efficiency of photovoltaic (PV) system based by PID controller," Int. Research Journal of Engineering and Technology, vol. 4, no. 5, pp. 2273-2277, May 2017.

[7] Y. Safan, S. Shaaban, and A. El-Sebah, "Hybrid control of a solar tracking system using SUI-PID controller," in Proc. of Sensors Networks Smart and Emerging Technologies (SENSET), BeirutLebanon, Sept. 12-14, 2017. DOI: 10.1109/SENSET.2017.8125035

[8] S. Hanwate, and Y. hote, "Design of PID controller for sun tracker system using QRAWCP approach," Int. J. of Computational Intelligence Systems, vol. 11, pp. 133-145, 2018. DOI: 10.2991/ijcis.11.1.11

[9] N. Tatu, and C. Alexandru, "Modeling and simulation of the tracking mechanism for a PV string," in Proc. of IEEE International Conference on Automation, Quality and Testing, Cluj-Napoca Romania, May 2427, 2012, pp. 428-433. DOI: 10.1109/AQTR.2012.6237748 
[10] C. Alexandru, "A novel open-loop tracking strategy for photovoltaic systems," The Scientific World Journal, vol. 2013, p. 12, 2013. DOI: $10.1155 / 2013 / 205396$

[11] J. Denavit, and R. Hartenberg, "A Kinematic notation for lower-pair mechanisms based on matrices," J. Applied Mechanics, vol. 22, pp. 215-221, 1955.

[12] R. Jazar, Theory of applied Robotics, Springer: New York: Springer, 2007.

[13] A. Fernández-Pizarro, and J. Beltrán-González, "Design of a PID controller to govern the movement of orientable solar panels," Undergraduate degree work, Dept. Mech. Eng., Universidad del Atlántico, Puerto Colombia, Colombia, 2017. (In Spanish)

[14] C. Crane, and J. Duffy, Kinematic analysis of robot manipulators, New York, NY, USA: Cambridge University Press, 2008.

[15] M. Spong, S. Hutchinson, and M. Vidyasagar, Robot dynamics and control, 2nd ed., New York, NY, USA: Wiley, 2004.

[16] L. Tsai, Robot analysis, the mechanics of serial and parallel manipulators, New York, NY, USA: John Wiley and Sons, 1999.

[17] Sun Earth Tools. (2017). Sun Position, [Online]. Available: http://www.sunearthtools.com/dp/tools/pos_sun.php

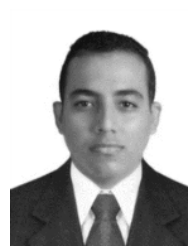

Andrés Fernández-Pizarro was born in Santo Tomás (Colombia) in 1992. He received his Mechanical Engineer degree from Universidad del Atlántico (Puerto Colombia, Colombia) in 2017. Since 2017, he works as a Maintenance Engineer at CSP Tubos 360 Company (Barranquilla, Colombia). His research interest includes design, modeling and control of mechanisms.

ORCID: http://orcid.org/0000-0003-2516-3829

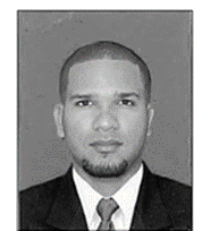

Jaison Beltrán-González was born in Barranquilla (Colombia) in 1987. He obtained his Electrician technical degree from SENA (Barranquilla, Colombia) in 2004. He obtained his Mechanical Engineer degree from Universidad del Atlántico (Puerto Colombia, Colombia) in 2017. He is currently working as Operations Engineer at Navarro Tovar S.A.S. (Barranquilla, Colombia). His research interest focuses in maintenance management and projects.

ORCID: http://orcid.org/0000-0001-9051-3253

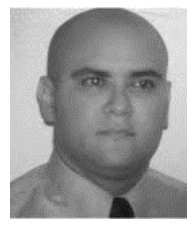

Javier A. Roldán-Mckinley. Former Professor of the City University of New YorkCUNY at LaGuardia Campus (2007-2011). He is currently a Full Time Faculty Researcher and Associate Professor of the Mechanical Engineering Program at Universidad del Atlántico (Puerto Colombia, Colombia). He earned his $\mathrm{PhD}$ degree in Mechanical Engineering from the Mechanical and Aerospace Department at University of Florida (Gainesville, USA) in 2007, majoring in Dynamics, Systems and Controls with concentration in Robotics Systems Design. He also holds a MSc degree with major in Machine Science, concentration in Mechanisms and Machine Theory through the Mechanical Engineering Department at University of Puerto RicoMayagüez (2003). He is a Mechanical Engineer from Universidad del Atlántico (Puerto Colombia, Colombia) since 2001. Professor Roldán research interest and courses are mainly related to Instrumentation, Controls, Machine Design, Automation and Robotics.

ORCID: http://orcid.org/0000-0003-1444-6460 